\title{
«Hören wir doch auf zu jammern»
}

\section{Das Interview führte: Matthias Scholer}

Online- und Printredaktor SÄZ

\author{
Feminisierung, Work-Life-Balance, Teilzeitstellen - Trends im Arbeitsmarkt machen \\ auch vor der Medizin nicht halt. Anstatt solche Entwicklungen zu kritisieren, kann \\ man auch mit innovativen Konzepten darauf reagieren. In einer kleinen Serie \\ möchten wir in loser Reihenfolge lösungsorientierte Ansätze vorstellen. Die Platt- \\ form «Rent A Senior Doc» des Hausarztes Bernhard Keller macht den Anfang.
}

\begin{abstract}
Sie haben vor vier Jahren Ihre Hausarztpraxis altershalber abgetreten. Bereuen Sie diesen Schritt?

Zuerst einmal war ich enorm froh, dass ich dank Praxisassistenz zwei Frauen gefunden habe, die meine Praxis übernommen haben. Damit wusste ich, dass meine Patientinnen und Patienten weiterhin gut versorgt werden. Was ich seither nicht vermisse, ist, eine eigene Praxis zu führen. Nach der Praxisübergabe genoss ich denn auch erstmal ein halbes Jahr Auszeit. Ich bewarb mich danach bei einer ärztlichen Hilfsorganisation und durfte über diese einige kurze, aber sehr intensive Einsätze in Ländern wie Bangladesch oder den Philippinen mitmachen. Dabei konnte ich unter den primitivsten Bedingungen, ganz auf mein Wissen und wenige Medikamente gestützt, bis zu hundert Patienten am Tag helfen. Diese Arbeit zeigte mir, dass meine
\end{abstract}

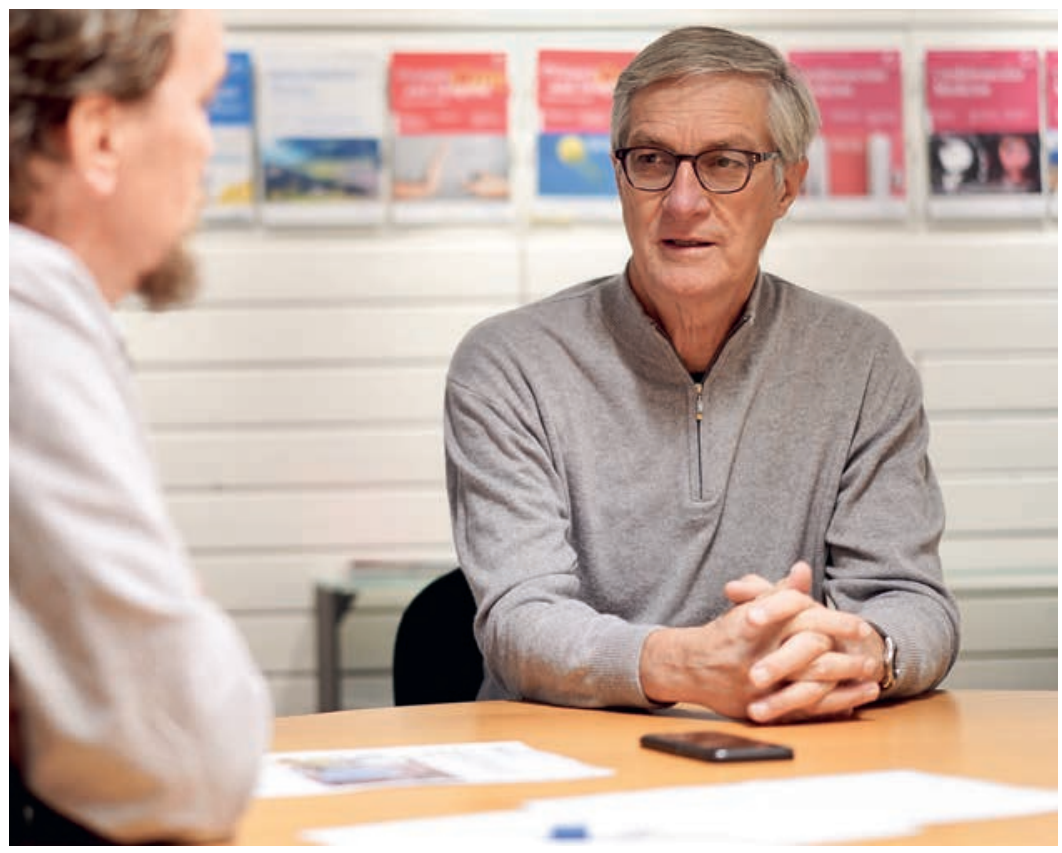

Bernhard Keller hilft aktiv mit, personelle Engpässe in Hausarztpraxen zu vermeiden.
Leidenschaft, «Medizin zu betreiben», noch nicht in Pension gegangen ist.

\section{Sie betreiben seit einiger Zeit die Website www. rentaseniordoc.ch. Was bezwecken Sie mit dieser Seite?}

Rent a Senior Doc ist eine Plattform, über die sich Hausärztinnen und Hausärzte, die temporäre Einsätze in ihrer Region suchen, und Praxen, die kurzfristig personelle Unterstützung benötigen, finden können. Und das ohne Vermittlungsgebühren, Agenturverträge oder sonstige Verpflichtungen mir gegenüber.

\section{Der Name der Plattform lässt darauf schliessen,} dass Sie vor allem pensionierte Ärztinnen und Ärzte suchen ...

Das Wort «Senior» bezieht sich mehr auf das Attribut «erfahren». Bei mir dürfen sich deshalb gerne auch Wiedereinsteigende melden und nicht nur Pensionierte. Die Grundbedingung ist, dass man genügend Berufserfahrung in der Grundversorgung mitbringt, um selbständig arbeiten zu können.

\section{Wie kommt Ihr Angebot an?}

Ich bekam viele wohlwollende Reaktionen seitens meiner Kolleginnen und Kollegen. Aber die Website ist noch ungewollt ein Geheimtipp. Das liegt hauptsächlich daran, dass ich die Plattform kaum bewerbe. Ich nehme an, die wenigsten wissen von diesem Angebot. Dann gibt es aber auch sicherlich viele Kolleginnen und Kollegen, die keine Lust mehr haben, in einer Praxis zu arbeiten. Bei anderen steigt mit jedem Jahr nach der Pensionierung die Hemmschwelle, zu praktizieren.

\section{Stehen Sie selber auch auf der Liste der Ärzte,} die sich als Verstärkung anbieten?

Selbstverständlich. Und ich habe auch schon in einigen Praxen ausgeholfen. 


\section{Und wie waren Ihre Erfahrungen?}

Durchwegs positiv. Meine eigene Praxis war in den letzten fünfzehn Jahren so stark ausgelastet, dass ich keine Patienten mehr aufnehmen konnte. Ich wünschte mir jedoch immer wieder, mich mit neuen, mir unbekannten Patienten auseinandersetzen zu dürfen. Diese Möglichkeit hatte ich nun während meiner Vertretungen. Ich bin überzeugt, dass diese Unbefangenheit sowohl für die einzelnen Patienten als auch die Praxiskollegen von Vorteil sein kann.

\section{Inwiefern?}

Wenn man einen Fall neu vorgestellt bekommt, geht man häufig mit einer anderen Perspektive an die Aufarbeitung als der Kollege, der sich schon länger damit beschäftigt. Zudem kommt es immer wieder vor, dass Patienten bei jemand Neuem eine andere Aussage machen, die für die Diagnosestellung oder die Therapiewahl entscheidend sein kann. Zu guter Letzt bringt eine solche Vertretung immer auch frischen Wind in die Kommunikation innerhalb der Ärzte einer Praxis.

\section{Und wie war die Akzeptanz seitens der Patienten, wenn da plötzlich ein unbekannter Arzt stand?}

In Gruppenpraxen ist das eigentlich nie ein Problem. Dort sind die Patienten Arztwechsel eher gewohnt. In Einzelpraxen kann das etwas schwieriger sein. Aber als Vertretung kümmert man sich meistens um Notfälle und chronische Fälle. Bei diesen Patienten ist die Akzeptanz, sich mit einem fremden Arzt auseinanderzusetzen, höher.

\section{Die Medizin entwickelt sich stetig weiter, und die Digitalisierung ist auch in der Grundversorgung ein Thema. Hatten Sie nie Angst, sich in einer Hausarzt- praxis nicht mehr zurechtzufinden?}

Doch. Aber wenn man eine Praxisvertretung annimmt, muss man auch bereit sein, sich eine gewisse Blösse zu geben. Es ist immer eine Herausforderung, sich in ein bestehendes Team einzugliedern, und man hofft, die Erwartungen zu erfüllen. In den insgesamt sechs Praxen, in denen ich schon Vertretungen gemacht habe, war beispielsweise das System des Führens und Ablegens der Krankengeschichten nie gleich geregelt. Von der noch rein handschriftlichen bis hin zur komplett digitalen Aktenführung habe ich alles angetroffen. Ich bin wahrlich ein PC-Novize und habe es trotzdem noch jedes Mal geschafft, die Daten korrekt einzulesen. Mittlerweile empfinde ich die vollelektronischen Lösungen als sehr hilfreich.
Die Mehrheit der pensionierten Ärztinnen und Ärzte verfügen wohl kaum mehr über eine Praxisbewilligung. Ein Problem?

Eigentlich nicht. Vor einer Vertretung reiche ich ein Gesuch um die Bewilligung für eine Praxisvertretung ein. Bislang wurde mir eine solche meist umgehend erteilt. Auf meiner Website habe ich übrigens unter der Rubrik «Checkliste» die für eine Praxisvertretung nötigen rechtlichen und versicherungstechnischen Punkte aufgeführt.

Ihre Initiative kann punktuell helfen, Versorgungsengpässe in Hausarztpraxen zu lindern. Wo müsste Ihrer Meinung nach der Hebel für eine langfristige Lösung angesetzt werden?

Wir müssen mehr Mediziner ausbilden. Es ist doch beschämend, dass wir so viele ausgebildete Medizinerinnen und Mediziner mit hohen Löhnen anlocken, die dann in ihren Ursprungsländern fehlen. Wir sollten das Geld besser in Ausbildungsplätze hierzulande investieren. Wenn ich lese, dass 70\% an Medizin interessierten jungen Menschen am Eignungstest scheitern, verspielen wir so Jahr für Jahr ein Riesenpotential an zukünftigen Ärztinnen und Ärzten.

Bedeuten mehr Studenten auch mehr Grundversorger? Natürlich entscheiden sich viele für eine Spezialisierung. Aber als Hausarzt ist man auf ein gutes Netz von Spezialistinnen und Spezialisten angewiesen. Zudem kann man den Studierenden während der Ausbildung den Beruf der Hausärztin, des Hausarztes auch näherbringen und die vielen guten Seiten dieses Betätigungsfeldes aufzeigen. Ich denke auch, dass der Trend weg von Einzelpraxen hin zu Gruppenpraxen die Attraktivität des Hausarztberufs stark erhöht, weil sich so die Arbeit auf mehr Schultern verteilen lässt und flexible Arbeitszeitmodelle möglich sind.

\section{Aber die Bezahlung der Hausärztinnen und Hausärzte ist doch schlecht? \\ Bei fast jeder Weiterbildung, die ich besuche, wird nach spätestens zehn Minuten über TARMED gesprochen. Hören wir doch auf, zu jammern! Uns geht es doch gut. Wir Hausärztinnen und Hausärzte haben alle ein eige- nes Dach über dem Kopf und mindestens ein Auto vor dem Haus! Und wir haben einen anspruchsvollen, aber auch sehr erfüllenden Beruf.}

Bildnachweis Tanja Kühnle 